\title{
Numerical study of water and heat transfer in unsaturated clay-loam soil
}

\author{
Nacer Lamrous*, Said Makhlouf, and Nora Belkaid \\ Mouloud Mammeri University, L.M.S.E. Laboratory, PO Box 17, RP 15000, Tizi-Ouzou, Algeria
}

Received 12 June 2013 / Accepted 13 November 2013 / Published online 10 March 2014

\begin{abstract}
This present study is the numerical estimation of the temperature distribution and the water content distribution underground soil under the Mediterranean climate type. We use as input data of ambient temperature, air humidity and solar radiation, average values during 10 years estimated from data supplied by the local meteorological station (Tizi-Ouzou, Northern Algeria, 36 $47^{\prime} 59^{\prime \prime}$, North latitude and $4^{\circ} 1^{\prime} 59^{\prime \prime}$, East longitude). A theoretical model, based on heat and water transfer equations, was established for an unsaturated soil submitted to the climatic conditions of this site. The mathematical model established in mono dimensional type, for a semi infinite transfer model, is based on Whitaker theory of heat and mass transfers in unsaturated porous medium (Withaker 1977, 1980) with the hypothesis that air pressure into soil porosity is equal to atmospheric pressure. The equations were discretized according to the finite volume method, which is more adapted for this type of problem, and were solved by the Newton-Raphson iterative method in the environment of Matlab software. The simulations have been done for two typical days (January 15 and May 15). Curves of temperature and water content evolutions in term of depth and time were obtained.
\end{abstract}

Key words: Heat and mass transfer, Soil temperature, Unsaturated porous soil, Simulation.

\section{Introduction}

Theoretical prediction of mass and heat transfer in an unsaturated porous medium is still complex. This is due to the discontinuity of these media and the diversity of the phenomena taking part in this process. It is also due to the difficulty of predicting the phenomenological coefficients to be introduced in the calculation programs, as reported by Moyne [7]. In this theoretical study, the mathematical model is based on Whitaker's approach $[12,13]$, and the technique of the representative elementary volume (REV, see Figures 1 and 2).

This allows us to assimilate the porous medium to a continued soil. The air and water mass conservation equations in both liquid and steam forms and the heat conservation equation are written down at the scale of pores, and then integrated in the domain of REV. We have developed a code of calculation and simulated the behaviour of the soil. In this program, the thermo physical characteristics such as density, heat capacity, absolute permeability, thermal conductivity and porosity have been measured in the LCTP (Central Laboratory of Public Works, Tizi-Ouzou). The meteorological conditions of the site are taken out of files where temperature and humidity, solar radiation and wind velocity during the period of ten years are consigned by meteorological station of Tizi-Ouzou.

\section{Basic mathematical model}

The variables that we have retained for the study of this problem are the moisture content $X$, the temperature $T$ along with the pressure $P$ of the gas phase constituted of a mixture of water steam and dry air. In order to be confined to the dominating phenomena and simplify a certain number of calculations, we adopt some main hypothesis such as:

- The deformations of the solid phase are neglected; thus its speed is zero;

- The local temperature is identical for the three phases;

- The liquid phase is incompressible;

- The constituents of the gas phase are expected to behave like perfect gas.

\subsection{Pore scale equations}

Before developing the conservation equations, we must introduce certain variables which intervene in this problem. In fact, the moisture content $X$ can be written as follows:

$$
X=\frac{m_{w}}{m_{s}}=\frac{\rho_{w} V_{w}+\rho_{v} V_{g}}{\rho_{s} V_{s}} .
$$




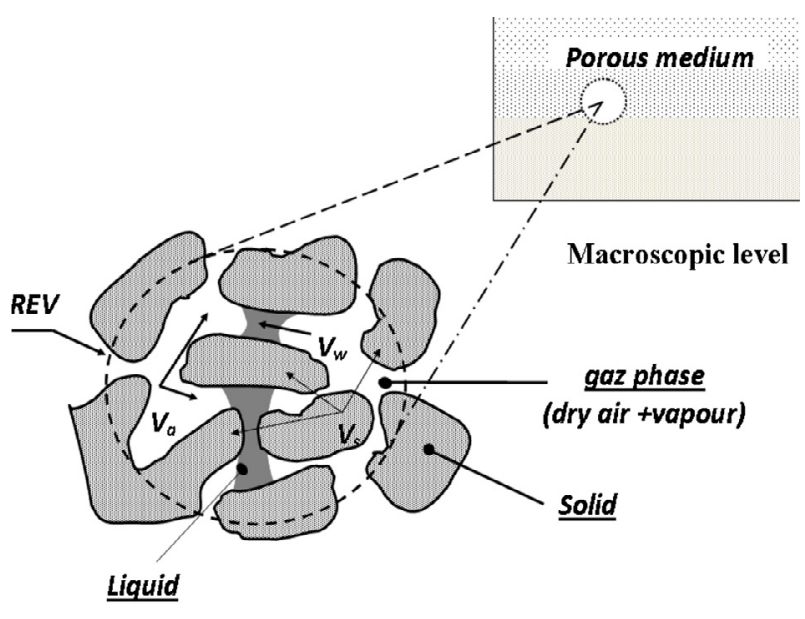

Pore level

Figure 1. Illustration of representative volume element (REV).

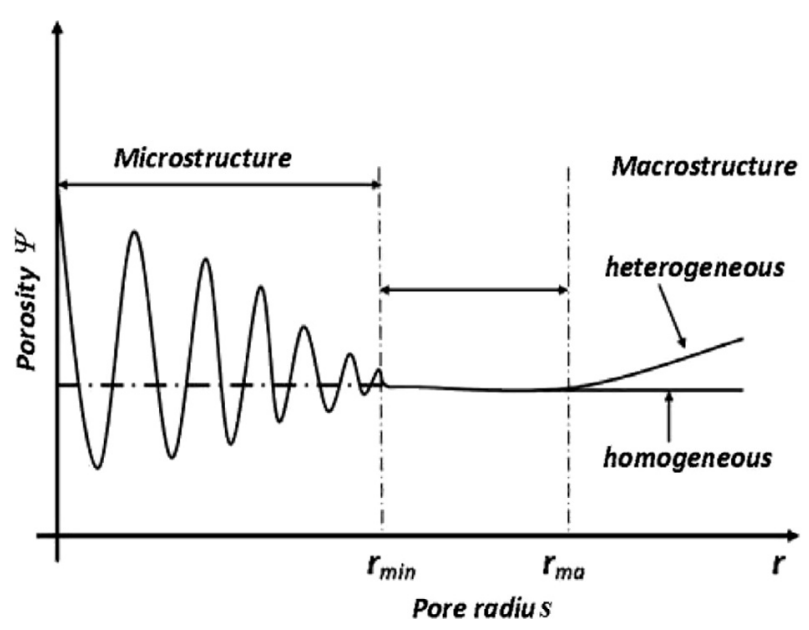

Figure 2. Porosity variation in porous medium at different scales.

We define also the volume fractions of water, gas and solid in the porous medium as follows:

$$
\varepsilon_{w}=\frac{V_{w}}{V} ; \quad \varepsilon_{g}=\frac{V_{g}}{V} ; \quad \varepsilon_{s}=\frac{V S}{V} .
$$

We obtain thus:

$$
X=\frac{\rho_{w} \varepsilon_{w}+\rho_{v} \varepsilon_{g}}{\rho_{s} \varepsilon_{s}} .
$$
liquid:

Saturation $S$ is the volume fraction of void space filled by

$$
S=\frac{V_{w}}{V_{w}+V_{g}}=\frac{V_{w}}{V-V_{s}} .
$$

The porosity $\psi$ of a porous material is the ratio of the total void or volume pore to the total volume of the material:

$$
\Psi=\frac{V_{w}+V_{g}}{V} .
$$

The relationship between moisture content and saturation is:

$$
X=\frac{\Psi \rho_{w}}{1-\Psi} S
$$

In these relations, $m, \rho$, and $V$ represent respectively mass, density and volume and the subscripts $a, g, s, v$ and $w$ are used to designate respectively dry air, gas, solid, vapour or water components.

The conservation equations of water, dry air and energy are written as follows:

$$
\begin{aligned}
& \frac{\partial}{\partial t}\left(\rho_{w} \varepsilon_{w}+\rho_{v} \varepsilon_{g}\right)+\nabla \cdot\left(\rho_{w} v_{w}+\rho_{v} v_{g}\right) \\
& \quad=\nabla \cdot\left[\rho_{g} D_{\text {eff }} \cdot \nabla\left(\frac{\rho_{v}}{\rho_{g}}\right)\right]
\end{aligned}
$$

$$
\frac{\partial}{\partial t}\left(\varepsilon_{g} \rho_{a}\right)+\nabla \cdot\left(\rho_{a} v_{g}\right)=\nabla \cdot\left[\rho_{g} D_{\text {eff }} \cdot \nabla\left(\frac{\rho_{a}}{\rho_{g}}\right)\right],
$$

$$
\begin{gathered}
\frac{\partial}{\partial t}\left(\varepsilon_{s} \rho_{s} h_{s}+\varepsilon_{w} \rho_{w} h_{w}+\varepsilon_{g} \rho_{v} h_{v}+\varepsilon_{g} \rho_{a} h_{a}\right) \\
+\nabla \cdot\left[\rho_{w} h_{w} v_{w}+\left(\rho_{v} h_{v}+\rho_{a} h_{a}\right) v_{g}\right]=\nabla \cdot\left[\rho_{g} h_{a} D_{\mathrm{eff}} \cdot \nabla \frac{\rho_{a}}{\rho_{g}}\right] \\
+\nabla \cdot\left[\rho_{g} h_{v} D_{\text {eff }} \cdot \nabla \frac{\rho_{v}}{\rho_{g}}\right]+\nabla \cdot\left(\lambda_{\text {eff }} \nabla T\right) .
\end{gathered}
$$

In addition to above conservation equations, gas and water velocities must be evaluated. Their expressions, given by the generalized law of Darcy [2] are written by neglecting the gravitational terms for gas and liquid phases, as follows:

$$
v_{w}=-\frac{K \cdot k_{w}}{\mu_{w}} \cdot \nabla P_{w} \text { and } \mathrm{v}_{g}=-\frac{K \cdot k_{g}}{\mu_{g}} \cdot \nabla P_{g} .
$$

Here $v, P, \varepsilon, h$ and $T$ are respectively the velocity, pressure, volume fraction, mass enthalpy and temperature of a considered porous media component. $D_{\text {eff }}$ and $\lambda_{\text {eff }}$ are the effective vapour diffusivity and effective thermal conductivity. They are obtained from the process of up scaling from pore scale transport equations to macroscopic equations [12]. $K$ and $k$ are the absolute and relative permeability respectively and $\mu$ is the dynamic viscosity.

\subsection{Boundary conditions}

In order to complete the conservation equations listed beforehand, we associate the following boundary conditions:

The temperature $T_{s}$, on the soil surface, is calculated by thermal balance on the assumption that the dominating exchanges are done within the atmosphere (conductive exchanges in the soil are neglected):

$$
\begin{aligned}
& (1-\alpha) R_{g}-\varepsilon \sigma_{B}\left(T_{s}^{4}-\left(T_{a}-6\right)^{4}\right)-h_{c}\left(T_{s}-T_{a}\right) \\
& \quad=L_{v} J_{w}
\end{aligned}
$$


Table 1. Average meteorological parameters of Tizi-Ouzou site, 2011.

\begin{tabular}{|c|c|c|c|c|c|c|c|c|c|c|c|c|}
\hline & Jan & Feb & Mar & Apr & $\mathrm{Ma}$ & Jun & Jul & $\mathrm{Au}$ & Sep & Oct & Nov & Dec \\
\hline Solar radiation $\left(\mathrm{w} \mathrm{m}^{-2}\right)$ & 472 & 541 & 624 & 671 & 633 & 640 & 620 & 641 & 586 & 506 & 453 & 438 \\
\hline Relative humidity (\%) & 84.8 & 65.5 & 64.5 & 60.8 & 54.5 & 47.8 & 42.0 & 46.2 & 53.9 & 56.9 & 63.1 & 65.1 \\
\hline
\end{tabular}

Table 2. Physical and thermal soil parameters.

\begin{tabular}{|c|c|c|c|c|c|}
\hline & $\begin{array}{c}\text { Density } \rho_{s} \\
\left(\mathrm{~kg} \mathrm{~m}^{-3}\right)\end{array}$ & $\begin{array}{c}\text { Thermal } \\
\text { conductivity } \lambda_{s} \\
\left(\mathrm{~W} \mathrm{~m}^{-1} \mathrm{~K}^{-1}\right)\end{array}$ & $\begin{array}{l}\text { Mass thermal } \\
\text { capacity } C_{p, s} \\
\left(\mathrm{~kJ} \mathrm{~kg}^{-1} \mathrm{~K}^{-1}\right)\end{array}$ & Porosity $\Psi(\%)$ & $\begin{array}{c}\text { Absolute } \\
\text { permeability } \\
\mathrm{Ka}\left(\mathrm{m}^{2}\right)\end{array}$ \\
\hline Value & 1515 & 1 & 1270 & 40 & $10^{-14}$ \\
\hline
\end{tabular}

where $T_{a}$ is the ambient temperature, $L_{v}$ is the latent heat of evaporation, $\alpha$ is the soil albedo and $R_{g}$ is the global solar flux.

$J_{w}$, the flux of water mass is described for convective drying by the boundary layer theory with Stefan correction:

$$
J_{w}=\frac{K_{E} M_{v}}{R T_{a}}\left(h_{\mathrm{r}} P_{v}^{\mathrm{sat}}\left(T_{s}\right)-P_{v}\left(T_{a}\right)\right)
$$

Here, $K_{E}$ is the mass transfer coefficient, $M_{v}$ is the vapour molar mass, $h_{\mathrm{r}}$ is relative humidity and $R$ is the universal gas constant. The superscript sat designate saturation state. The conductive heat flux is zero because the temperature undergoes very slight variations at a certain depth, whereas the moisture content of the soil is maximal and equal to the moisture content of the saturated soil.

\subsection{Parameters of simulation}

To study the behaviour of soil submitted to climatic conditions of Tizi-Ouzou, we rely on meteorological data collected at the local station on typical days of a 12-month year. As initial conditions, we have taken the measured values of temperatures and for moisture content, we choose a linear profile. Here are included the curves of annual evolution of the monthly average of temperatures of both air and soil noted down in Tizi-Ouzou. Table 1 shows the average monthly values of global solar radiation and relative humidity measured at meteorological local station.

To simulate heat and water transfers in soil, we use the thermo physical characteristics provided by the LCTP laboratory and shown in Table 2.

It is known that the effective conductivity coefficient is independent of pore size distribution. As heat conduction occurs in all phases in parallel, the heat flux or thermal conductivity contributions must be weighted according to their respective volume fractions of the phases. The serial model of conductivities of different components is appropriate in these conditions. Thus, if the contribution of gas is neglected, the effective thermal conductivity can be computed as:

$$
\lambda_{\mathrm{eff}}(S, T)=(1-\psi) \lambda_{S}+S \psi \lambda_{w}(T),
$$

$\Psi$ is the porosity of soil, $\lambda_{s}$ is soil thermal conductivity, $S$ is saturation and $\lambda_{w}$ is the water conductivity. According to
Van Genuchten, cited by Lefebvre [6], the relative permeability of liquid water is:

$$
k_{w}(S)=\left(1-\left(1-S^{1 / m}\right)^{m}\right)^{2} \sqrt{S} .
$$

For the capillary pressure, we retained the expression of Van Genuchten (1980), written in terms of the effective saturation $S_{\text {eff }}$ and which introduces two empirical parameters, $\beta$ and $m$.

$$
\begin{aligned}
P_{c}\left(S_{\text {eff }}\right) & =\left(\left(S_{\text {eff }}\right)^{(-1 / m)}-1\right)^{(1-m)} / \beta \text { with } S_{\text {eff }} \\
& =\left(\theta-\theta_{r}\right) /\left(\theta_{m}-\theta_{r}\right) .
\end{aligned}
$$

For a silty soil, we took the values recommended by Calvet [1]: $\quad \beta=0.0115 \mathrm{~Pa}^{-1} ; \quad m=0.5169 ; \theta_{m}=0.52 \mathrm{~kg} \mathrm{~m}^{-3}$ et $\theta_{\mathrm{r}}=0.218 \mathrm{~kg} \mathrm{~m}^{-3}$.

For the relative humidity of air inside the soil, we will take again the expression obtained from fitting curves of the sorption isotherm [10], reported by Hong $\mathrm{Vu}$ [4], considering:

$$
H_{r}=\frac{P_{v}}{P_{v}^{\text {sat }}(T)}=\left\{\begin{array}{ll}
1 & \text { if } X>X_{\text {irr }} \\
\frac{X\left(2-\frac{X}{X_{\text {irr }}}\right)}{X_{\text {irr }}} & \text { if } X \leq X_{\text {irr }}
\end{array},\right.
$$

$X_{\text {irr }}$ is the irreducible water content defined as the value of moisture content above which the water is free in the pores.

The saturating vapour pressure is calculated according to the relation given by [11]:

$$
P_{v}^{\mathrm{sat}}(T)=133.32 \exp [18.584-3984.2 /(233.426+T)] .
$$

The binary diffusion coefficient of vapour in air is calculated from equation given by Schirmer [5]:

$$
\delta_{v a}(T, P)=2.2610^{-5}\left(T / T_{R}\right)^{1.81} P_{R} / P_{g},
$$

where, $T_{R}$ and $P_{R}$ are reference temperature and pressure, respectively.

The surface tension in porous medium is expressed according to [11]:

$$
\sigma(T)=-1.310^{-7} T^{2}-1.5810^{-4} T+0.07606 .
$$




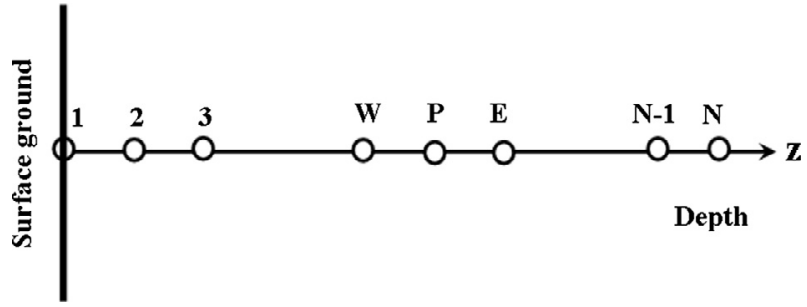

Figure 3. Control volume mesh.

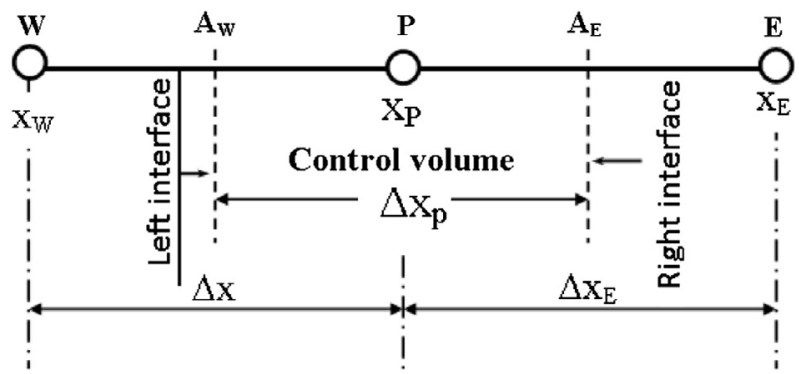

Figure 4. Normal control volume element for one-dimensional problems.

\subsection{Discretization of the equations}

For the discretization of these equations, the volume control method was used as recommended by Patankar [9]. Integrating the conservation equation of water over the control volume element and time duration, we obtain for each node $P$ as shown in Figures 3 and 4:

$$
\begin{aligned}
& \int_{t}^{t+\Delta t} \int_{x_{W}}^{x_{E}}\left[\varepsilon_{s} \rho_{s} \frac{\partial X}{\partial t}+\frac{\partial}{\partial t}\left(\varepsilon_{g} \rho_{v}\right)\right] \mathrm{d} V \mathrm{~d} t-\int_{t}^{t+\Delta t} \\
& \quad \times \int_{x_{W}}^{x_{E}} \nabla J_{w} \mathrm{~d} V \mathrm{~d} t \\
& \quad=0 .
\end{aligned}
$$

Then:

$$
\begin{aligned}
& \frac{\left(\Delta x_{W}+\Delta x_{E}\right)}{2 \Delta t}\left[\left(\varepsilon_{s} \rho_{s} X+\varepsilon_{g} \rho_{v}\right)_{P}^{t+\Delta t}-\left(\varepsilon_{s} \rho_{s} X+\varepsilon_{g} \rho_{v}\right)_{P}^{t}\right] \\
& \quad+\left[\left(J_{w}\right)_{E}^{t+\Delta t}-\left(J_{w}\right)_{W}^{t+\Delta t}\right] \\
& \quad=0 .
\end{aligned}
$$

The water flux $J_{w}$ from east and west boundary surfaces of control volume can be expressed as:

$$
\begin{aligned}
\left(J_{w}\right)_{e}^{t+\Delta t}= & \left(\rho_{w} \frac{K k_{w}}{\mu_{w}}\right)_{A_{E}}^{t+\Delta t} \frac{\left(P_{w}\right)_{E}^{t+\Delta t}-\left(P_{w}\right)_{P}^{t+\Delta t}}{\Delta x_{E}} \\
& +\left(\rho_{g} D_{\text {eff }}\right)_{A_{E}}^{t+\Delta t} \frac{\left(y_{v}\right)_{E}^{t+\Delta t}-\left(y_{v}\right)_{P}^{t+\Delta t}}{\Delta x_{E}}
\end{aligned}
$$

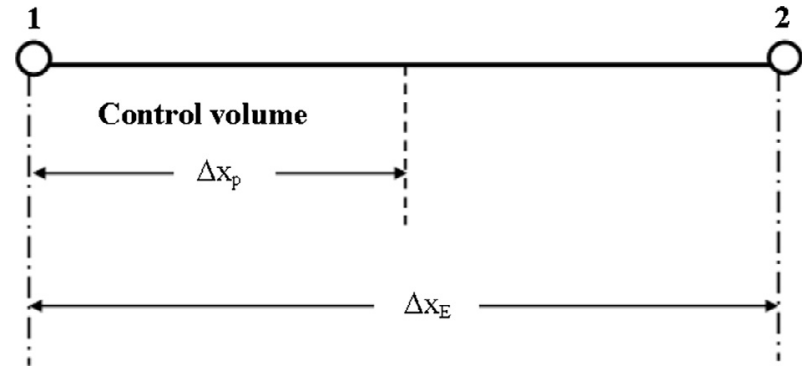

Figure 5. Control volume for the 1 st element.

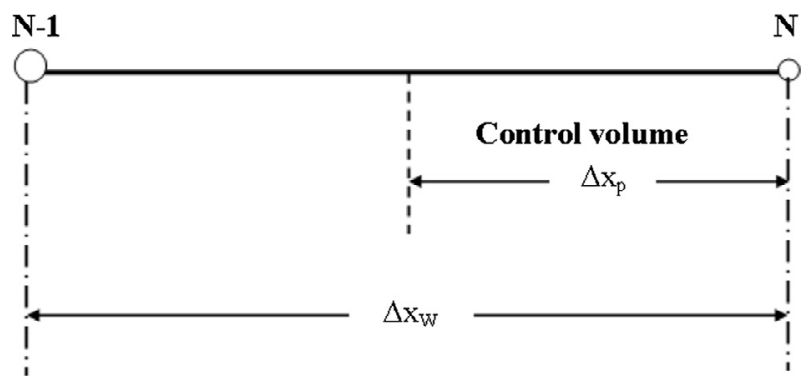

Figure 6. Control volume for the $n$th element.

$$
\begin{aligned}
\left(J_{w}\right)_{w}^{t+\Delta t}= & \left(\rho_{w} \frac{K k_{w}}{\mu_{w}}\right)_{A_{W}}^{t+\Delta t} \frac{\left(P_{w}\right)_{P}^{t+\Delta t}-\left(P_{w}\right)_{W}^{t+\Delta t}}{\Delta x_{W}} \\
& +\left(\rho_{g} D_{\text {eff }}\right)_{A_{W}}^{t+\Delta t} \frac{\left(y_{v}\right)_{P}^{t+\Delta t}-\left(y_{v}\right)_{W}^{t+\Delta t}}{\Delta x_{W}}
\end{aligned}
$$

For the 1st and the $N$ th element (cf. Figures 5 and 6), the discrete version of water conservation writes:

$$
\begin{aligned}
& \frac{\left(\Delta x_{E}\right)}{2 \Delta t}\left[\left(\varepsilon_{s} \rho_{s} X+\varepsilon_{g} \rho_{v}\right)_{1}^{t+\Delta t}-\left(\varepsilon_{s} \rho_{s} X+\varepsilon_{g} \rho_{v}\right)_{1}^{t}\right] \\
& \quad+\left[\left(J_{w}\right)_{A_{E}}^{t+\Delta t}-\left(J_{w}\right)_{1}^{t+\Delta t}\right] \\
& \quad=0
\end{aligned}
$$

$$
\begin{aligned}
& \frac{\left(\Delta x_{W}\right)}{2 \Delta t}\left[\left(\varepsilon_{s} \rho_{s} X+\varepsilon_{g} \rho_{v}\right)_{N}^{t+\Delta t}-\left(\varepsilon_{s} \rho_{s} X+\varepsilon_{g} \rho_{v}\right)_{N}^{t}\right] \\
& \quad+\left[\left(J_{w}\right)_{N}^{t+\Delta t}-\left(J_{w}\right)_{A_{W}}^{t+\Delta t}\right] \\
& \quad=0
\end{aligned}
$$

The conservation equation of energy is discretized in a similar way as that of water:

$$
\begin{aligned}
& \frac{\left(\Delta x_{W}+\Delta x_{E}\right)}{2 \Delta t}\left[\Psi_{P}^{t+\Delta t}-\Psi_{P}^{t}\right]+\left[\left(J_{e}\right)_{A_{E}}^{t+\Delta t}-\left(J_{e}\right)_{A_{W}}^{t+\Delta t}\right] \\
& \quad=0
\end{aligned}
$$

where:

$$
\begin{aligned}
\Psi_{P}^{t+\Delta t} & =\left(\overline{\rho C_{p}}\left(T-T_{R}\right)+\varepsilon_{g} \rho_{v} \Delta h_{v 0}\right)_{P}^{t+\Delta t} \text { et } \Psi_{P}^{t} \\
& =\left(\overline{\rho C_{p}}\left(T-T_{R}\right)+\varepsilon_{g} \rho_{v} \Delta h_{v 0}\right)_{P}^{t},
\end{aligned}
$$




$$
\begin{gathered}
\left(J_{e}\right)_{A_{E}}^{t+\Delta t}=\left(T_{A_{E}}^{t+\Delta t}-T_{\mathrm{ref}}\right)\left[\left(C_{a} \rho_{g} D_{\mathrm{eff}}\right)_{A_{E}}^{t+\Delta t}\right. \\
\frac{\left(y_{a}\right)_{E}^{t+\Delta t}-\left(y_{a}\right)_{P}^{t+\Delta t}+\left(C_{v} \rho_{g} D_{\mathrm{eff}}\right)_{A_{E}}^{t+\Delta t}}{\Delta x_{E}}+\left(\rho_{w} C_{w} \frac{K k_{w}}{\mu_{w}}\right)_{A_{E}}^{t+\Delta t} \\
\times \frac{\left(y_{v}\right)_{E}^{t+\Delta t}-\left(y_{v}\right)_{P}^{t+\Delta t}}{\Delta x_{E}}+\left(\Delta h_{v 0} \rho_{g} D_{\mathrm{eff}}\right)_{A_{E}}^{t+\Delta t} \\
\left.\times \frac{\left(P_{w}\right)_{E}^{t+\Delta t}-\left(P_{w}\right)_{P}^{t+\Delta t}}{\Delta x_{E}}\right]+\left(\lambda_{\mathrm{eff}}\right)_{A_{E}}^{t+\Delta t} \frac{(T)_{E}^{t+\Delta t}-(T)_{P}^{t+\Delta t}}{\Delta x_{E}} \\
\times \frac{\left(y_{v}\right)_{E}^{t+\Delta t}-\left(y_{v}\right)_{P}^{t+\Delta t}}{\Delta x_{E}} \\
\frac{\left(y_{a}\right)_{P}^{t+\Delta t}-\left(y_{a}\right)_{W}^{t+\Delta t}+\left(C_{v} \rho_{g} D_{\mathrm{eff}}\right)_{A_{E}}^{t+\Delta t}}{\Delta x_{W}} \\
\times \frac{\left(y_{v}\right)_{P}^{t+\Delta t}-\left(y_{v}\right)_{W}^{t+\Delta t}}{\Delta x_{W}}+\left(\rho_{w} C_{w} \frac{K k_{w}}{\mu_{w}}\right)_{A_{W}}^{t+\Delta t} \\
\left.\times \frac{\left(P_{w}\right)_{P}^{t+\Delta t}-\left(P_{w}\right)_{W}^{t+\Delta t}}{\Delta x_{W}}\right]+\left(\Delta h_{v 0} \rho_{g} D_{\mathrm{eff}}\right)_{A W}^{t+\Delta t} \\
\times \frac{\left(y_{v}\right)_{P}^{t+\Delta t}-\left(y_{v}\right)_{W}^{t+\Delta t}}{\Delta x_{W}}+\left(\lambda_{\mathrm{eff}}\right)_{A W}^{t+\Delta t} \frac{(T)_{P}^{t+\Delta t}-(T)_{W}^{t+\Delta t}}{\Delta x_{W}}
\end{gathered}
$$

For element 1: $\frac{\Delta x_{E}}{2 \Delta t}\left[\Psi_{1}^{t+\Delta t}-\Psi_{1}^{t}\right]$

$$
+\left[\left(J_{e}\right)_{A_{E}}^{t+\Delta t}-\left(J_{e}\right)_{1}^{t+\Delta t}\right]=0 .
$$

For element $N: \frac{\Delta x_{E}}{2 \Delta t}\left[\Psi_{N}^{t+\Delta t}-\Psi_{N}^{t}\right]$

$$
+\left[\left(J_{e}\right)_{N}^{t+\Delta t}-\left(J_{e}\right)_{A_{W}}^{t+\Delta t}\right]=0 .
$$

The mass conservation equation of the dry air is not used because the total pressure is considered as constant in the soil since the dry air pressure can be deduced from the partial pressure of the vapour.

\section{Numerical results and interpretations}

We represent here the curves of time evolution of temperature and moisture levels and their evolution as a function of depth, starting first at the free surface of the soil, then deeper up to $3 \mathrm{~m}$. Figure 7 shows the evolution of average month temperature measured at ground surface and in ambient air under shedder.

Figures 8 and 9 show the evolution of the soil temperature at free surface level and at different depths: $0.0 ; 0.1 ; 0.3$ and $0.5 \mathrm{~m}$. Both curves reveal clearly that the variations of the outdoor climatic conditions during the day affect only the upper layers of the soil, nearly down to $50 \mathrm{~cm}$ in the case of the characteristics of the soil on which we carried out the study.

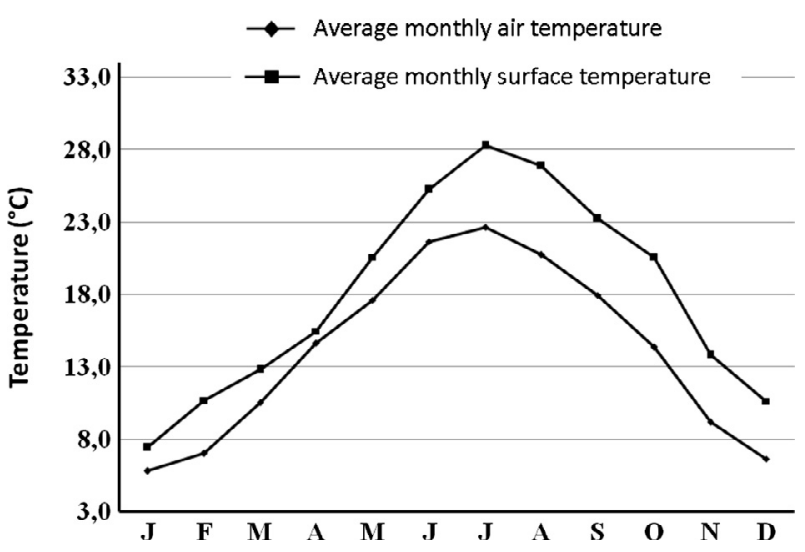

Figure 7. Average monthly air and surface soil temperature.

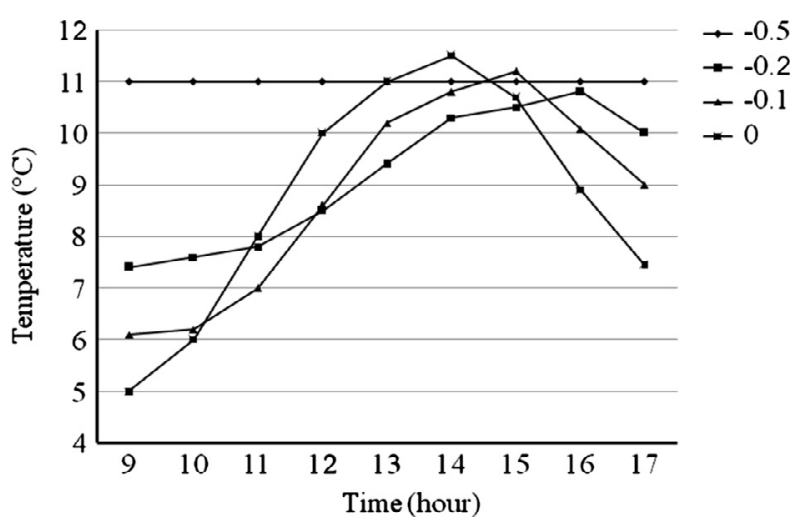

Figure 8. Horary evolution of the soil temperature (January 15).

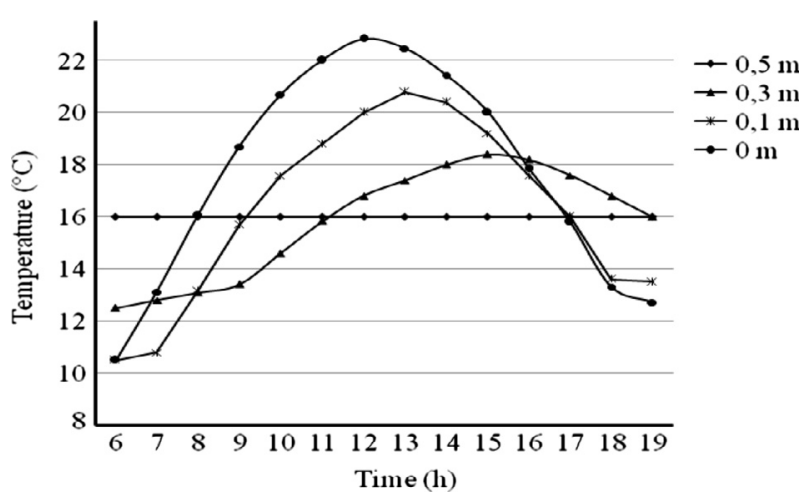

Figure 9. Horary evolution of the soil temperature (May 15).

The surface temperature is attenuated in depth and is affected by a progressive dephasing, associated with the inertia of the soil. Thus, during a typical day of May, the maximum temperature reaches $23{ }^{\circ} \mathrm{C}$ at the free surface level, at $12 \mathrm{~h} 30 \mathrm{mn}$, and decreases to $18.2{ }^{\circ} \mathrm{C}$ at $30 \mathrm{~cm}$ depth, with a gap of $3 \mathrm{~h}$. This phenomenon is also reproduced in January with lower temperature maxima and a more important time difference in the afternoon. These results as well as the evolution of the temperature within this free surface - the top layer - are confirmed by previous works on this theme $[3,8]$. At the lower layers, the 


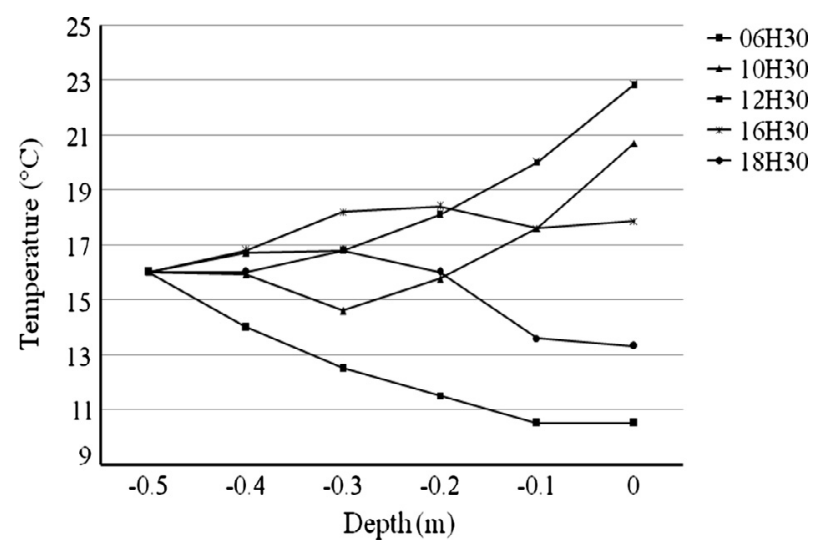

Figure 10. Temperature evolution in terms of depth (May 15).

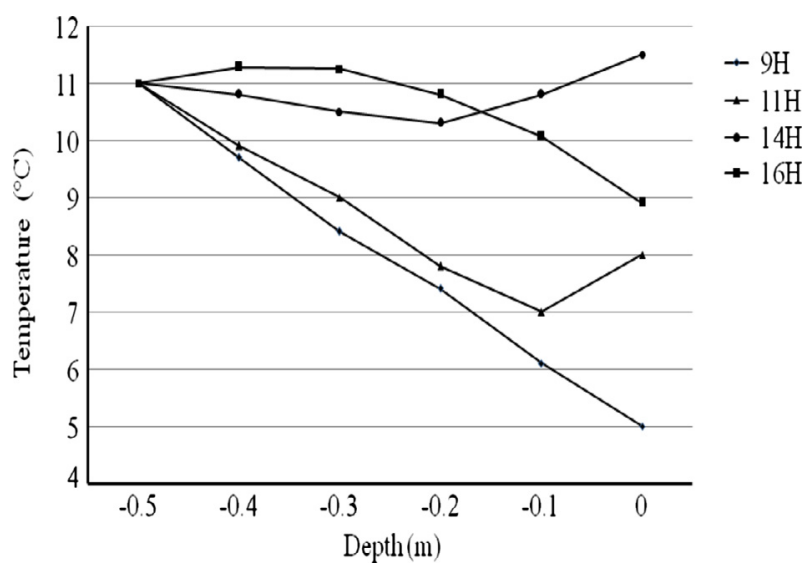

Figure 11. Temperature evolution in terms of depth (January 15).

temperatures are constant during the day (we have noticed this in some measures carried out in situ). As we go deeper over $3 \mathrm{~m}$, the temperature goes down to the monthly average soil temperature $\left(17^{\circ} \mathrm{C}\right.$ in May).

Figure 10 (for May 15) and Figure 11 (for January 15) show the evolution of the temperature in depth at different moments of the day. Thus for May 15 at 6 h 30, the temperature is lower on the surface and increases as we go deeper until $0.5 \mathrm{~m}$ where

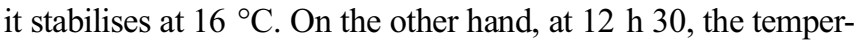
ature is higher on the surface and decreases until $0.5 \mathrm{~m}$ of depth where it stabilises again at the same value of $16^{\circ} \mathrm{C}$. For January 15 (see Figure 11), the temperature is greater on the surface for $11 \mathrm{~h}$ and $14 \mathrm{~h}$. It decreases in the first centimeters and increases again until it reaches $11{ }^{\circ} \mathrm{C}$ at $50 \mathrm{~cm}$ depth.

Figures 12 and 13 show the evolution of water content down to $50 \mathrm{~cm}$ of the upper ground layer. This parameter is slightly affected by the climatic variation during the day. However, we notice a slight rising of humidity on the surface at the hottest moment of the day. But this phenomenon is observed only in the first $10 \mathrm{~cm}$.

When we are interested by the water content evolution in greater depths, we observe that it does not change during all the day time. Figure 14 shows that water content raises until it reaches the saturation at $3 \mathrm{~m}$ depth while keeping the initial linear profile (condition is set up at this limit). The study of this

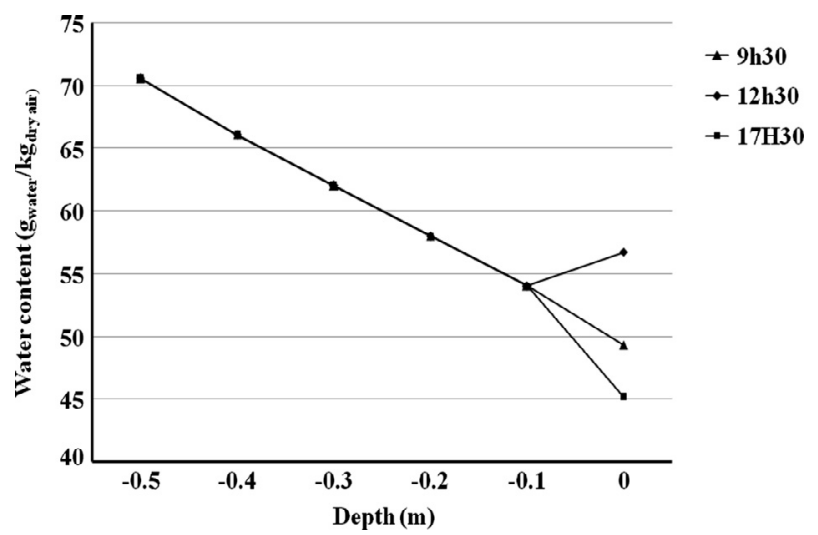

Figure 12. Water content evolution in terms of depth (May 15).

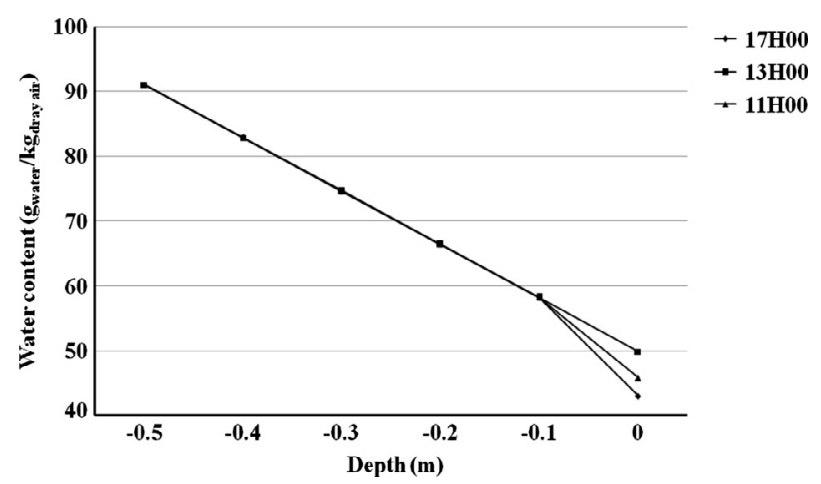

Figure 13. Water content evolution in terms of depth (January 15).

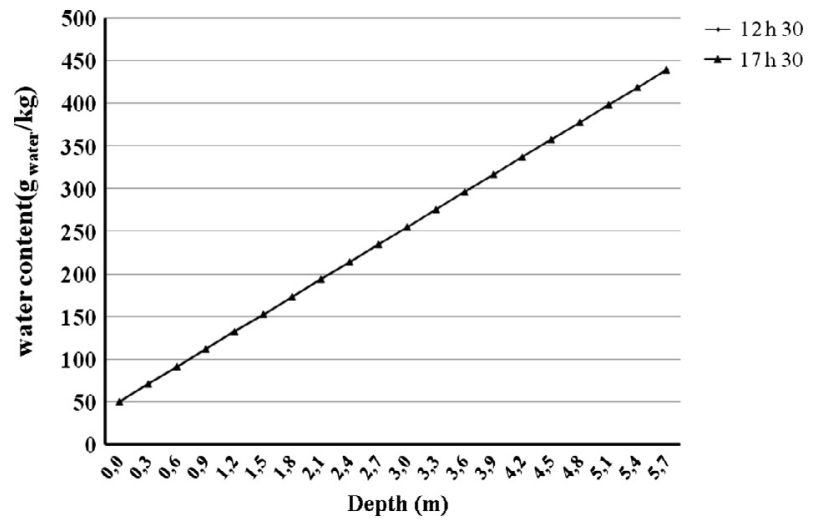

Figure 14. Water content in soil depth.

phenomenon must be conducted over a longer duration, such a complete season.

\section{Conclusion}

This theoretical study allows the reconstitution of the thermal and hydrous behaviour of unsaturated silty soil in real conditions at the scale of a day and as a function of depth. The results obtained by theoretical model are satisfactory and confirmed by the referred literature. However, the validation via 
experiment is necessary to validate again the value of the numerous parameters and the thermo physical characteristics which intervene in this problem. The computer code developed here can be a valuable tool in several domains such as agricultural science to help choose the plants and depth of the root system and the bioclimatic engineering and facilities such as underground heat exchangers in Canadian wells. With soil temperature and water content data, we can evaluate the heat flux and water flux through the soil layers. Thus, the water flux by capillary suction can be estimated for soil at known porosity and saturation.

\section{References}

1. Calvet R. 2003. Le sol: propriétés et fonction. Volume 2. Phénomènes physiques et chimiques. Applications agronomiques et environnementales. France agricole. Dunod 2003.

2. De Vries DA, Kruger AJ. 1966. On the value of the diffusion coefficient of water vapour in air. Proceeding du Colloque International du CNRS n ${ }^{\circ} 160$ : Phénomènes de transport avec changement de phase dans les milieux poreux ou colloïdaux. CNRS éditeur, 18 au 20 Avril 1966. p. 61-72.

3. Gao Z, Bian L, Hu Y, Wang L, Fan J. 2007. Determination of soil temperature in an arid region. Journal on Arid Environments, 71, 157-168.

4. Hong Vu T. 2006. Influence of pore size distribution on drying behaviour of porous media by a continous model, $\mathrm{PhD}$-engineer. Otto Von Guericke University of Magdeburg, Germany.
5. Krischer O, Kast W. 1992. Die wissenschaftlichen Grundlagen der Trocknungstechnik, erster Band, dritte Auflage. Berlin: Springer.

6. Lefevbre R. 2003. Ecoulement Multiphasique en Milieux Poreux. Hiver 2003.

7. Moyne C. 1987. Transferts couplés chaleur-masse lors du séchage: prise en compte du mouvement de la phase gazeuse. Doctorat thesis of Polytechnical National Institut of Lorraine, France.

8. Nebbali R. 2008. Modélisation de la dynamique du climat interne distribué dans une serre de culture. Doctorat thesis of Franche-Comté University, France.

9. Patankar SV. 1980. Numerical Heat Transfer and Fluid Flow. Hemisphere Publ. Co: New York.

10. Perre P, Turner IW. 1999. A 3-D version of transPore: a comprehensive heat and mass transfer computational model for simulating the drying of porous media. International Journal of Heat and Mass Transfer, 42, 4501-4521.

11. Vargaftik NB, Vinogradov JK, Yargin VS. 1996. Handbook of Physical Properties of Liquids and Gases: Pure Substances and Mixtures, 3rd edn. Begell House: New York.

12. Whitaker S. 1977. Simultaneous heat, mass, and momentum transfer in porous media: a theory of drying. Advances in Heat Transfer, 13, 119-203.

13. Whitaker S. 1980. Heat and mass transfer in granular porous media, in Advances in Dryng 1. Mujumdar AS, Editor. Hemisphere Publ. Co: Washington, DC. p. 23-61.

Cite this article as: Lamrous N, Makhlouf S \& Belkaid N: Numerical study of water and heat transfer in unsaturated clay-loam soil. Int. J. Simul. Multisci. Des. Optim., 2014, 5, A19. 